Chapter 13

\title{
Calibrating Biosensors in Flow-Through Set-Ups: Studies with Glucose Optrodes
}

\author{
K. Kivirand, M. Kagan and T. Rinken \\ Additional information is available at the end of the chapter \\ http://dx.doi.org/10.5772/54127
}

\section{Introduction}

The research and development of selective analytical systems, not requiring pre-treatment of samples and rapidly providing information in real time, is receiving increasing attention in various areas of human life (Chen et al, 2011;Palmisano et al, 2000;Maestre et al, 2005;Jia et al, 2004;Surareungchai et al, 1999;Tsai \& Doong, 2005;Gülce et al, 2002;Akin et al, 2011;Mishra et al, 2012). For example, as environmental pollution poses a serious threat to the human health, there is an urgent demand to monitor pollutants and initiate appropriate environmental pollution treatment in the real time course. In the field of food quality control, the product quality and healthiness are the main factors, influencing customer satisfaction. The number of food processing and manufacturing mistakes can be minimized with risk assessment and continuous checking of the production process, e.g. in dairy farms, it is necessary to control the quality of raw milk on site in order to detect the presence of the residues of different antibiotics and other potentially harmful compounds before loading milk into the dairy production process.

On-site monitoring requires enhanced sensitivity, selectivity, rapidity, and ease of operation of the analytical equipment, which should provide reliable continuous information in realtime and demonstrate sufficient stability of action. Biosensors fulfil all the above-mentioned requirements and have already been applied in clinical diagnostics, food quality control, forensic chemistry, environmental monitoring and other areas (Castillo et al, 2004;Reder-Christ \& Bendas, 2011;Kivirand \& Rinken, 2011).

According to the IUPAC definition, a biosensor is a self-contained, integrated receptortransducer device, which is capable of providing selective quantitative or semi-quantitative analytical information and which uses a biological recognition element (bio-receptor) and a transducer in direct special contact (Thevenot et al, 2001). A biosensor consists of three parts: 
(1) the sensitive biological element (such as tissues, microorganisms, cell receptors, enzymes, antibodies, nucleic acids, etc.); (2) the transducer or the detector element (physiochemical, optical, piezoelectric, etc.) that transforms the signal, resulting from the interaction between the analyte and the biological element into another signal that can be measured and quantified; and (3) associated electronics or signal processors that are primarily responsible for the display of the results in a user-friendly way. All these three parts are associated with an information management system. The principle of biosensors is shown in figure 1 .

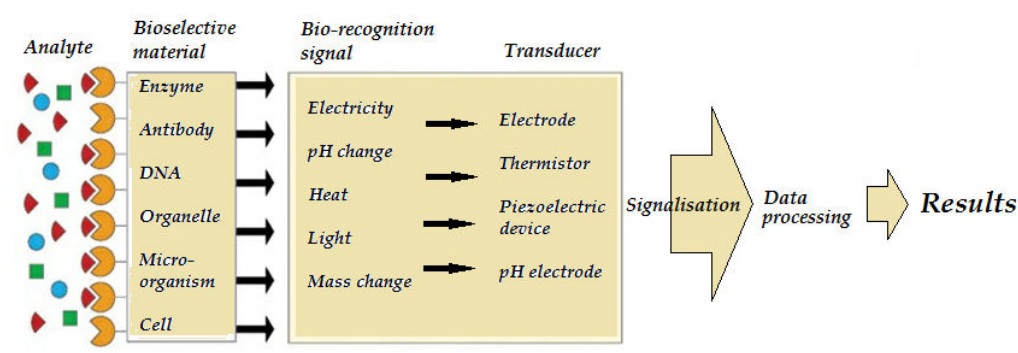

Figure 1. The principle of biosensor systems.

Enzyme-based biosensors technology relies upon the natural specificity of a given enzymatic protein to act selectively on a target analyte or group of analytes. Enzymes are catalysts bearing some excellent properties that may permit to perform the most complex chemical processes under the most benign experimental and environmental conditions. Enzymebased biosensors have emerged as a valuable technique for qualitative and quantitative analysis of a variety of target analytes. Although biosensors based on other biorecognition elements are rapidly progressing, enzyme biosensors are still the ones most frequently used for practical applications and as model systems in scientific studies. There are several advantages of enzyme-based biosensors: a known reaction mechanism, a stable bio-renewable source of material and possibilities to modify the catalytic properties or substrate specificity by means of genetic engineering or to use catalytic amplification by the modulation of enzyme activity with respect to the target analyte (Castillo et al, 2004;Hu et al, 2011).

For the implementation at industrial scale, the properties of enzymes have to be improved further, as soluble enzymes should be immobilized for their multiple utilizations. The immobilization of enzymes and the choice of an insoluble carrier are important features in designing the biorecognition part of enzyme-based biosensors. Various immobilization strategies can be envisioned: adsorption, entrapment, covalent cross-linking or affinity (Sassolas et al, 2012;Cao, 2005;Mateo et al, 2007;Gibson, 1999). In some cases, enzyme immobilization protocols are also based on the combination of several immobilization methods: for example, an enzyme can be pre-immobilized on a carrier by adsorption, affinity or covalent bonding before further entrapment into a porous polymer. Biosensors based on immobilized enzymes have good operational and storage stability, high sensitivity and selectivity, good reproducibility and additionally, as enzyme immobilization reduces the time of enzymatic 
response, these biosensors can be easily used in continuous-flow and flow-through systems (Sassolas et al, 2012; Yang et al, 2010;Castillo et al, 2004;Cao, 2005).

The other moiety of a biosensor is the signal transduction system, which can be based on the measurement of electrochemical, magnetic, piezoelectric, thermometric or optical signals (Mehrvar \& Abdi, 2004;Mello \& Kubota, 2002;Sarma et al, 2009;Castillo et al, 2004). Among the above-mentioned systems, fibre-optic sensors are gradually achieving popularity. In comparison with electrochemical transducers, they do not consume any analytes and are insensible to electrical or magnetic interference (in fact, the oxygen detection capability has been demonstrated on single luminescent molecules) (D.R.Walt, 2006;Leung et al, 2007). Fluorescence measurements can be used whenever a fluorescent analyte is detected. Naturally fluorescent compounds are not common in biosensor development and the technique is usually applied in combination with artificially labelled compounds. Fluorescence is applied, for instance in biosensors with oxidase-type enzymes, which catalyze the consumption of oxygen, resulting the decrease in the luminescent signal of a fluorescent dye attached to the surface of an optical fibre. Besides a direct detection of the analyte of interest, the optical biosensor format may also involve indirect detection through optically labelled probes. Optical transducers may detect changes of absorbance, luminescence, polarization or refractive index and can be adapted for the assembly of different enzyme-substrate systems. For example, using a fibre-optic sensor assay which senses $\mathrm{pH}$ changes, Viveros et al. have demonstrated a rapid detection of organophosphates (insecticides and potent neurotoxins) (Viveros et al, 2006) and Bidmanova et al. developed an enzyme-based fibre-optic biosensor by co-immobilization of purified enzyme and a fluorescent $\mathrm{pH}$ indicator (Bidmanova et al, 2010). Polster et al. have immobilized enzymes onto an array of optical fibers for use in the simultaneous detection of penicillin and ampicillin. These biosensors employ an interferometric technique based on following the shifts in the reflectance spectrum, caused by the $\mathrm{pH}$ changes of the solution during the penicillinase - catalyzed hydrolysis of the analytes, penicillin and ampicillin (Polster et al, 1995).

The incorporation of an optical fibre into a biochemical sensor results in several advantages: (1) numerous optically based methods are available for chemical analysis, as almost every chemical analyte can be determined by measuring its spectroscopic properties; (2) fibres can be used to transmit light over long distances; (3) fibres have a multiplex capability (because they can guide light of different wavelengths at the same time and in different directions, multiple- or single-analyte monitoring in single locations can be performed with a single central unit); (4) fibres can be used in harsh environments and are immune to electric or magnetic interferences, and so can be safer than electrochemical biosensors; (5) fibres can be easily miniaturized at low cost (6) fibres can be made biocompatible and thus used for in-vivo measurements; (7) a light guide can carry more information than electric wire; and (8) the temperature-dependence of the fibre is lower than that of common electrodes (Marazuela \& Moreno-Bondi, 2002).

Some drawbacks of optical-fibre sensors can limit their applicability: (1) interference of ambient light, although this can be avoided by use of suitable light isolation or modulated light sources; (2) background absorbance or fluorescence of the fibre itself; (3) long response times 
if mass transfer to the reagent phase is needed; and (4) limited availability of optimized commercial accessories for use with optical fibres (Marazuela \& Moreno-Bondi, 2002).

Fibre-optic sensors are a perspective replacement of wide-spread Clark-type amperometric sensors for the detection of oxygen, although the application of oxygen measuring is hindered due to their sensitivity to oxygen fluctuations in natural samples (Li \& Walt, 1995). To overcome the problem of the variability of oxygen concentrations, a two-sensor (or dual-sensor) approach with a reference sensor included in the system can be used. Pasic et al. have used a microdialysis-based glucose-sensing system based on a fibre-optic hybrid sensor (Pasic et al, 2006; Pasic et al, 2007). They used a reference oxygen optrode to detect and compensate response changes caused by side events, like bacterial growth, temperature fluctuations or failure of the peristaltic pump. The constructed sensor was evaluated in vitro using a 3-day continuous testing. As a result, they found that all glucose readings were clinically accurate and acceptable. With the purpose of analyzing complex biological samples without the need for any sample pre-treatment, Chen et al. developed a thermal based flow injection biosensor system, where a reference column was used to detect the non-specific thermal response (Chen et al, 2011). This sensor system was used to detect urea and lactate in non-standard milk products (such as lactose free milk). They found that when using that kind of biosensing systems, it was not necessary to remove the interfering compounds during milk analysis. The sensitivity and accuracy of the analysis were in the ranges required by the dairy industry.

One of the key problems of real-time measurements is the calibration of the measuring system and the management of data acquisition to obtain the results as quickly as possible. It is common in biosensor studies that the only information used, is the steady-state output (or the presumed 95\% of it, the value of $T_{95}$ ) (Baker \& Gough, 1996). Unfortunately, the determination of the value of $T_{95}$ is often imprecise because of the difficulties in estimating the attainment of steady state - thus there is an urgent need for exact modelling of processes taking place in different set-ups of experimental measurements (Baker \& Gough, 1996;Li \& Walt, 1995; Lammertyn et al, 2006;Baronas et al, 2011). Measurements in continuous-flow systems require additional consideration of the flowing effects, both laminar and turbulent, in the biosensor output signal. The nature of the flow profile of the plug of solution introduced into the flowing stream of carrier solution is normally affected mainly by laminar flow. The resistance between solution and the wall of the tubing causes the solution to travel slower near the wall of the tubing. However, some reports demonstrate that laminar flow in the small tubing does not have much effect on distortion of the zone to the point that affects the accuracy of kinetic studies (Konermann, 1999;Zhou et al, 2003;Hartwell \& Grudpan, 2012).

The utilization of biosensors in continuous-flow manifolds allows samples to be manipulated or modified as required for the execution of various operations such as separation, automatic dilution or pre-concentration, or some kind chemical or biochemical reaction prior to the final detection step (Hansen, 1996). It is also necessary to distinguish clearly between continuous-flow biosensing (referred to as "sensor system") systems and flowthrough biosensors. The primary difference between these two systems lies in whether or not the detection of the analyte of interest is performed simultaneously with other ana- 
lytical steps (chemical reaction, separation, or both) in the continuous system. Thus in a continuous-flow sensor system, the biochemical reaction takes place before the sample has reached the flow cell for detection, while the flow-through biosensors involve the development of either the overall process or only the last step of the biochemical reaction in the flow cell (Hansen, 1996).

The properties of a continuous-flow biosensor must meet the requirement of being able to follow the maximal anticipated concentration fluctuations within a specific acceptable error (Baker \& Gough, 1996). In the flow systems the biosensor contacts the substrate for a short time only. When the analyte disappears, a buffer solution swills the enzyme surface, reducing the substrate concentration at this surface to zero. Because of (analyte) remaining in the enzyme membrane substrate, the mass diffusion as well as the reaction still continues for some time even after the disconnection of the biosensor and substrate (Baronas et al, 2002). Compared to a batch system, the flow system present the advantages of the reduction in analysis time allowing a high sample throughput and the possibility to work with small volumes of the substrate (Baronas et al, 2002;Baronas et al, 2011). The flow arrangement also presents a wide response range and high sensitivity. While modelling a biosensor in a flowing system, it is of crucial importance to take into consideration the external diffusion limitations, because of the mass transport outside the enzyme region (Baronas et al, 2011;Ivanauskas \& Baronas, 2008;Baronas et al, 2002).

Recently, the interest of constructing the fibre-optic flow biosensing systems is growing and these are mostly made for glucose monitoring (Pasic et al, 2007; Pasic et al, 2006; Zhu et al, 2002;Akin et al, 2011). Usually glucose oxidase is selected as a model enzyme in biosensing systems because of its low cost, stability and high solubility in different medium. Glucose oxidase is an enzyme which catalyzes the oxidation of $\beta$-D-glucose by molecular oxygen to $\delta$-gluconolactone, which subsequently hydrolyzes to gluconic acid and hydrogen peroxide (Bankar et al, 2009). The enzyme is also of considerable commercial importance, as it is used in the removal either of glucose or oxygen from food products and in the production of gluconic acid. The most important application of glucose oxidase is as a molecular diagnostic tool. The enzyme is used in biosensors for the quantitative determination of D-glucose in various samples of natural origin, such as body fluids, foodstuff, beverages, and fermentation products (Bankar et al, 2009).

The aim of the present research was to study the modelling of a biosensor response and to propose optional biosensor calibration parameters in a flowing medium. We used a glucose optrode, which was a dual-sensor system, enabling to eliminate the fluctuations in the initial dissolved oxygen concentration, temperature and fluidic flow. The system consisted of two oxygen optrodes, one covered with glucose oxidase-containing nylon thread and the other with a similar, but "blank" thread, which were placed into isolated parallel flow channels. Glucose biosensor was selected for these studies as the bioactive compound of this biosensor; enzyme glucose oxidase is a well-characterized robust enzyme of high stability and selectivity towards glucose. The effect of the speed of the flow on different calibration parameters, obtained from the transient phase of the biosensor signal, was studied. 


\section{Materials and experimental procedures}

Glucose oxidase (GOD, EC 1.1.3.4. from Aspergillus niger, 17300 U/g protein) was obtained from Sigma. All other reagents used in the study were of analytical grade. Glucose stock solutions were prepared in phosphate buffer $(\mathrm{PB})(\mathrm{pH} 6.50, I=0.1 \mathrm{M})$ and allowed to mutarotate overnight at $37^{\circ} \mathrm{C}$; glucose working solutions were prepared immediately before use.

\subsection{Enzyme immobilization}

Glucose oxidase was immobilized onto nylon-6,6 threads, used as a carrier, according to previously published protocol with some minor modifications. Nylon is a perfect carrier for enzyme immobilization, because it is inert, hydrophilic and mechanically strong. However, its inertness prevents enzyme binding without a specific treatment (Isgrove et al, 2001;Segura-Ceniceros et al, 2006;Sassolas et al, 2012;Nan et al, 2009). Hence, activation of nylon is essential in immobilizing of an enzyme. One of the possibilities to activate nylon surface is by $O$-alkylation with dimethyl sulfate (DMS). Thus, pieces of nylon thread with a length of $100 \mathrm{~cm}$ were immersed into $98 \%(\mathrm{w} / \mathrm{w})$ dimethyl sulfate at $50^{\circ} \mathrm{C}$ for $10 \mathrm{~min}$; washed thoroughly at first with ice-cold methanol and after that with $0.1 \mathrm{M} \mathrm{PB}(\mathrm{pH}$ 6.50). Thereafter, the threads were immersed into $12.5 \%$ glutaraldehyde solution (in $0.1 \mathrm{M} \mathrm{PB}, \mathrm{pH} 6.50$ ) for $1 \mathrm{~h}$ at room temperature. Glutaraldehyde (GA) was used as a linker between the activated carrier and enzyme (Betancor et al, 2006;Pahujani et al, 2008). The threads were washed with $0.1 \mathrm{M}$ $\mathrm{PB}$ (pH 6.50) and incubated overnight at $4^{\circ} \mathrm{C}$ in GOD solution (100 U/ml) (Scheme 1) (Kivirand \& Rinken, 2009). Finally, the threads were thoroughly washed and stored in a 0.1 M PB (pH 6.50) at $4^{\circ} \mathrm{C}$ until further use.

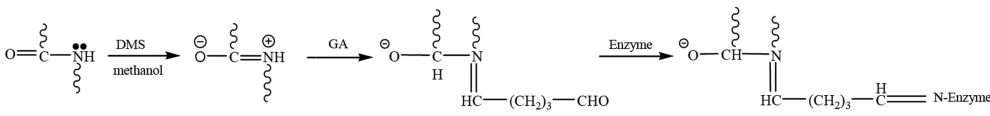

The GOD-containing threads kept at least $80 \%$ of their initial enzymatic activity for 35 days at $37^{\circ} \mathrm{C}$. The thread's activity was controlled before each series of measurements and the sensor output corrected according to the actual activity of the enzyme.

\subsection{Biosensor system}

Glucose oxidase catalyzes the oxidation of $\beta$-D-glucose by dissolved oxygen causing a decrease of dissolved oxygen concentration in the reaction medium:

$$
\beta-D \text {-glucose }+\mathrm{O}_{2} \rightarrow \mathrm{GOD} \text { - gluconic acid }+\mathrm{H}_{2} \mathrm{O}_{2}
$$

The applied oxygen optrode was constructed in the Institute of Physics at the University of Tartu. This sensor was based on measuring the oxygen-induced phosphorescence quenching of Pd-tetraphenylporphyrin molecules, encapsulated into thermally aged polymethyl 
methacrylate (PMMA) film, covering the cylindrical surface of a $30 \mathrm{~mm}$ long PMMA optical fibre with the diameter of $1 \mathrm{~mm}$ (Õige et al, 2005;Jaaniso et al, 2005). The dissolved oxygen concentration was calculated automatically with the help of Stern-Volmer relationship, using original software Oxysens 2.0.

For the preparation of a glucose optrode, $18 \mathrm{~cm}$ of GOD-containing thread was cut and coiled around the oxygen-sensitive surface of the oxygen optrode (Fig.2) (Kivirand et al, 2011). If the activity of the thread dropped below $80 \%$ of its initial activity, the GOD-containing thread was replaced. The reference optrode was covered with $18 \mathrm{~cm}$ of „blank“ thread.

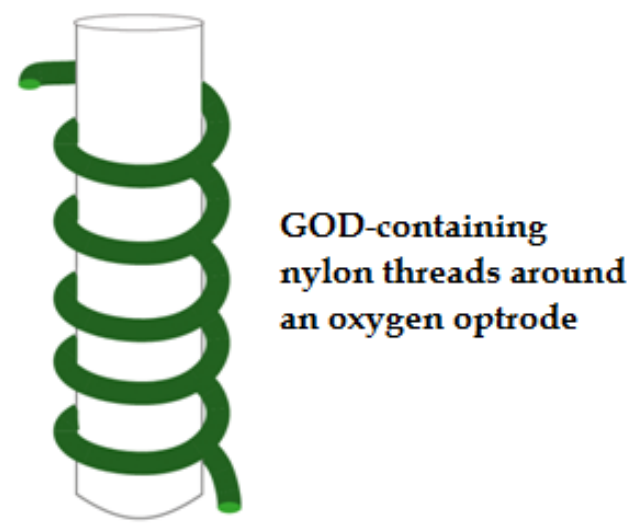

Figure 2. Construction of the biosensor: glucose oxidase-containing thread coiled spirally around an oxygen optrode.

The glucose and the reference optrodes were placed into identical and parallel isolated channels of the measuring cell. A schematic cross-section of the cell system used in the studies is presented in Fig.3. The flow channels were $50 \mathrm{~mm}$ long and with the diameter of 3 $\mathrm{mm}$. All measurements were carried out at flow speed varying from 0 to $5.1 \mathrm{~cm} / \mathrm{sec}$ at $37 \pm 0.02^{\circ} \mathrm{C}$. A peristaltic pump was used to deliver samples through the system and to wash the system; the temperature was stabilized by a specially constructed oven. To minimize temperature fluctuations, the flow tubing was going through the oven for 10 times before entering the measuring cell, making the temperature very stable, although slightly increasing the analysis time. The stabilization of the temperature was really important, as the analytical performance of the biosensor was anticipated to be greatly dependent on temperature (Pasic et al, 2007;Peedel \& Rinken, 2012). At temperature $37^{\circ} \mathrm{C}$ no enzyme denaturation could be detected and this temperature was used to gain the highest biosensor sensitivity. All measurements at different flow rates and different substrate concentrations were carried out in $0.1 \mathrm{M} \mathrm{PB}$ at $\mathrm{pH} 6.50$ (the oxygen saturation concentration at $37^{\circ} \mathrm{C}$ is $6.7 \mathrm{mg} / \mathrm{ml}$ ). 


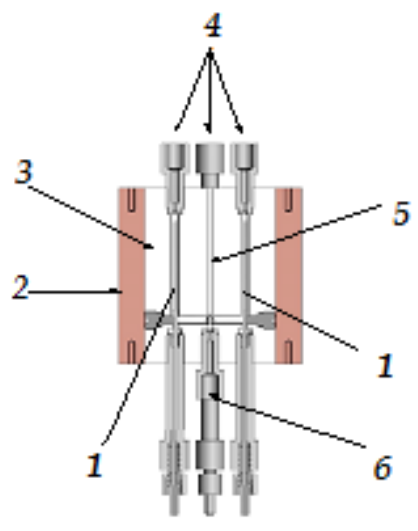

Figure 3. Schematic cross-section of the measuring cell 1 - glucose and oxygen optrodes, covered with nylon thread; 2 - cylindrical messing oven for the stabilization of temperature $\left( \pm 0.02^{\circ} \mathrm{C}\right) ; 3$ - measuring cell with flow channels; 4 outflows; 5 - temperature sensor; 6 -inflow.

In case the biosensor signal parameters were studied in a standing liquid, the glucose assays were injected at the speed of $1.1 \mathrm{~cm} / \mathrm{sec}$. After each measurement the system was washed with $0.1 \mathrm{M} \mathrm{PB}$ ( $\mathrm{pH}$ 6.50) until the sensor signals reached their initial values. The sensor output signal was recorded with the interval of $1 \mathrm{sec}$.

\subsection{Data processing}

The change of oxygen concentration was found as the difference between the signals of glucose and reference optrodes and normalized to bring the data from different sensors onto a common scale. From the reaction transient phase data, we calculated the total signal change parameter (at $t \rightarrow \infty$ ) using the earlier - proposed biosensor dynamic model, taking into account the ping-pong mechanism of enzyme kinetics, diffusion phenomena and the inertia of the signal transduction system (Rinken \& Tenno, 2001). According to this model, the normalized oxygen concentration $c_{\mathrm{O}_{2}}(t) / c_{\mathrm{O}_{2}}(0)$ during the bio-recognition process in a biosensor is expressed as a 3-parameter function of time $t$ :

$$
\frac{c_{\mathrm{O}_{2}}(t)}{{c_{\mathrm{O}_{2}}}(0)}=A \exp (-B t)+(1-A)-2 A \sum_{n=1}^{\infty}(-1)^{n} \frac{\tau_{s}}{n^{2} / B-\tau_{s}}\left[\exp (-B t)-\exp \left(-n^{2} \frac{t}{\tau_{s}}\right)\right]
$$


where $c_{\mathrm{O}_{2}}(t)$ is the biosensor output current at time moment $t ; c_{\mathrm{O}_{2}}(0)$ is the output current at the start of the reaction; $t$ is time.

The parameter $A$ is a complex coefficient, corresponding to the total possible biosensor signal change at the steady-state and parameter $B$ is the initial maximal slope of process curve; both parameters $A$ and $B$ depend hyperbolically on substrate concentration; $\tau_{s}$ is the time constant of the transducer's response (Rinken \& Tenno, 2001). Parameters $A, B$ and $\tau_{s}$ are all independent on each other. According to the applied model, the total signal change parameter $A$ is expressed as

$$
A=\frac{k_{\text {cat }}^{*}[E]_{\text {total }} c_{s}^{\text {bulk }}}{k_{\text {diff }}^{\mathrm{O}_{2}} K_{\mathrm{O}_{2}} K_{s}+\left(k_{\text {cat }}^{*}[E]_{\text {total }}+k_{\text {diff }}^{\mathrm{O}_{2}} K_{\mathrm{O}_{2}}\right) c_{\text {bulk }}}
$$

where $k_{c a t}^{*}$ is the apparent catalytic constant of the reaction; $[E]_{\text {total }}$ is the overall concentration of the immobilized enzyme; $k_{\text {diff }}^{\mathrm{O}_{2}}$ is the apparent diffusion constant of the oxygen; $K_{\mathrm{O}_{2}}$ is the dissociation constant of the enzyme-oxygen complex; $K_{s}$ the dissociation constant for the enzyme-substrate complex; and $c_{b u l k}$ is the substrate concentration in solution.

Additionally, from the transient phase data, collected between 20 to 60 seconds from the start of the reaction, the apparent maximal speed parameter of the reaction was calculated and used for the biosensor calibration. The starting moment of the reaction was determined experimentally for the particular measuring system: due to the length of the tubing the probe reached the optrodes after a time interval dependent on the flow speed. The values of all points on biosensor calibration curves are the results of at least 3 parallel measurements.

\section{Results}

\subsection{Output of the biosensor system}

The oxygen optrodes acting as oxygen transducers were employed to measure the rate of oxygen consumption in the enzymatic oxidation reactions. At fixed oxygen concentration, the response of the reference oxygen sensor is virtually constant with increasing glucose concentration, while the response of the glucose sensor decreases due to consumption of oxygen during glucose oxidation. The difference between the reference and the glucose sensor responses corresponds to the glucose concentration. Some examples of the signal curves of glucose and reference optrodes are shown in Fig. 4 (A). 

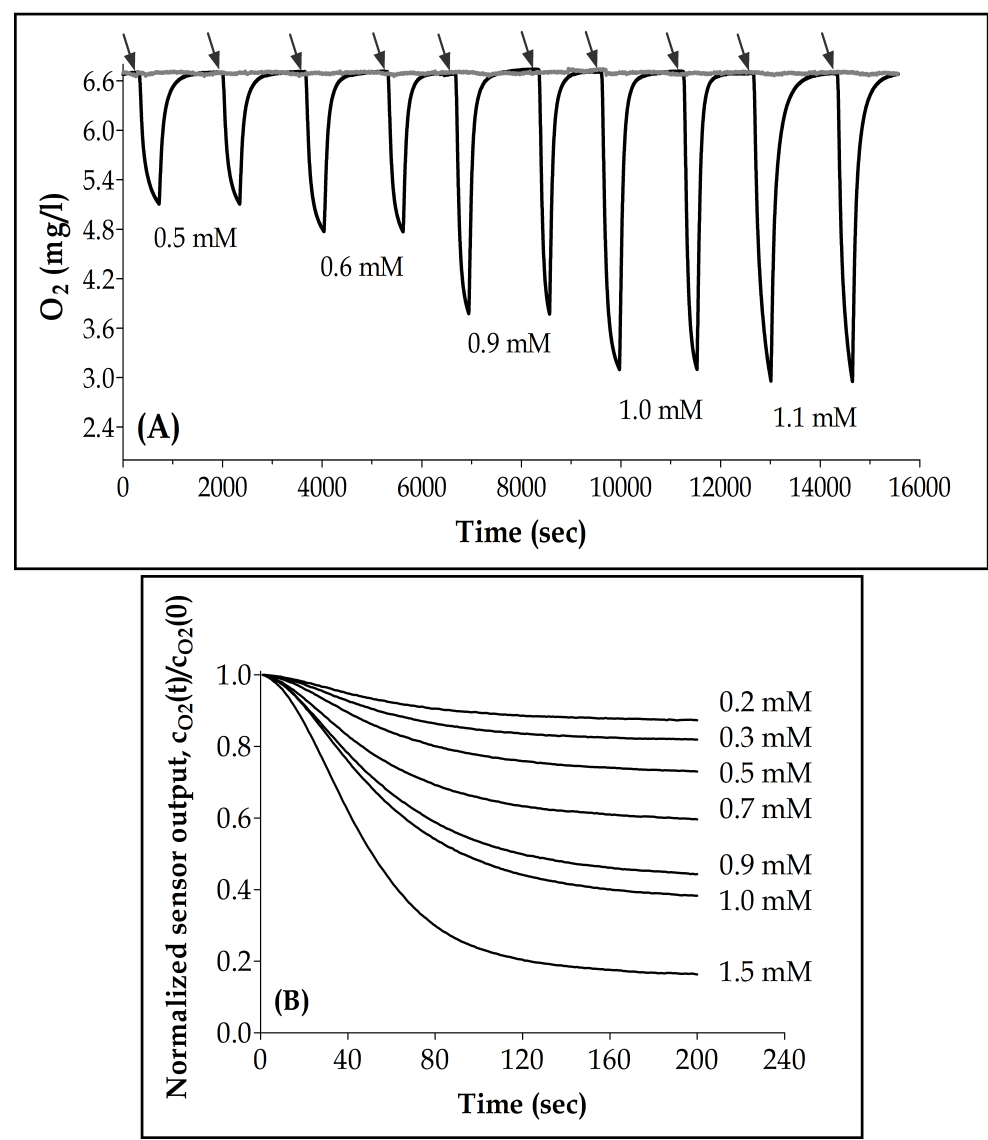

Figure 4. (A): Oxygen concentration responses obtained with the glucose oxidase optrode for different concentrations of glucose solution: - glucose biosensor response (black line); - reference optrode response (grey line) (at $37^{\circ} \mathrm{C}$ at flow rate $1.1 \mathrm{~cm} / \mathrm{sec}$ in a $0.1 \mathrm{M}$ phosphate buffer of $\mathrm{pH} 6.50$ ). Arrows indicate the substrate adding time. (B): Normalized sensor outputs at different glucose concentrations (at $37^{\circ} \mathrm{C}$ at flow rate $1.1 \mathrm{~cm} / \mathrm{sec}$ in $0.1 \mathrm{M}$ phosphate buffer of $\mathrm{pH} 6.50)$.

For the detection of the starting moment of the bio-recognition reaction, the dependence of the time gap between the injection of the probe into the tubing and the probe front reaching the optrodes on the speed of the flow was determined. For the particular measuring cell, it was linearly dependent on the speed of the flow as expected:

$$
t=103.30-(36.05 \pm 0.95) v
$$


where $t$ is the lag period and $v$ is the speed of the flow. Based on the value of this lag period for every measured flow speed, the biosensor data collected during this lag period was extracted from the databank, used for the calculation of the signal parameters at different glucose concentrations.

The response signal of the reference oxygen sensor was stable and its fluctuations did not exceed $1 \%$ of the working range of the sensor at any measured glucose concentration and flow speed. Still, to eliminate all potential experimental noise, the difference between the signals of the reference and the glucose sensor response was used to determine the normalized output of the system. An example of the normalized biosensor output curves at $v=1.1$ $\mathrm{cm} / \mathrm{sec}$ at different glucose concentrations are shown on Fig. 4 (B). These curves were used for the determination of the calibration parameters of the biosensor.

\subsection{System regeneration}

To use the bio-sensing system for real-time analysis, it is necessary to regenerate the system as quickly as possible. Regeneration involves passing a background flow of fluid without reactive components through the flowing system. The speed of cleaning of the flow system depends on the flow rate and slightly on the substrate concentration analyzed, as expected (Fig. 5).

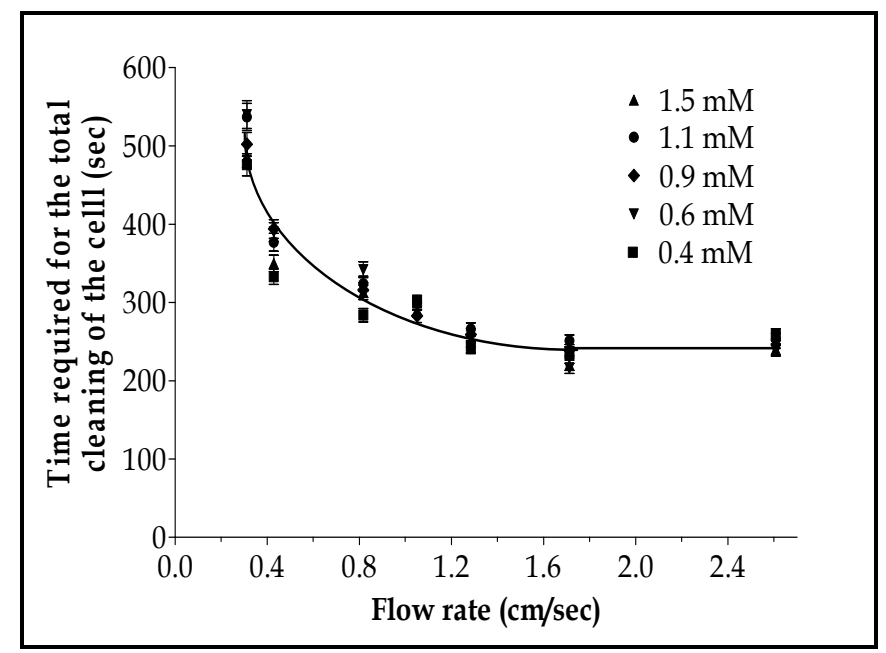

Figure 5. The speed of the cleaning of the biosensing system at different flow rates. Measurements were performed at $37^{\circ} \mathrm{C}$ in a $0.1 \mathrm{M}$ phosphate buffer $(\mathrm{pH} 6.50)$. The values of all points are the results of at least 3 parallel measurements. 
The flow rate was varied between 0.3 to $5.1 \mathrm{~cm} / \mathrm{sec}$. At lower flow rates $(0.3$ to $1.3 \mathrm{~cm} / \mathrm{sec})$ the system regeneration time increased by increasing of the flow rate. With further increase of the flow rate, the regeneration time became independent on the flow rate, which could be explained with the limits substrate diffusion in the GOD-containing threads. From Fig. 5, it can be seen that the regeneration time was also dependent on the substrate concentration. At lower flow rates $(0.3$ to $1.1 \mathrm{~cm} / \mathrm{sec})$ the dependence was clearly seen - increasing the substrate concentration the regeneration time also increased. At higher flow rates the regeneration time did not depend on the substrate concentration any more. From these results it could be concluded that the minimum required flow rate for system regeneration was at least $1.1 \mathrm{~cm} / \mathrm{sec}$. At this flow rate, the time for cleaning the system was 4.5 to $5 \mathrm{~min}$. Studies to minimize the regeneration time required, are ongoing.

\subsection{Calibration of the biosensor at different flow rates}

The flow rate in the system affected the values of the reaction parameters and thus the sample throughput, biosensor sensitivity and detection limit. The choice of optimal flow rate is the presumption of obtaining accurate and reliable results in flow-through biosensor setups. At low flow rates, the apparent speed of the enzyme - catalyzed reaction, registered with a biosensor, is smaller than at high flow speeds, but the steady state signal can be calculated more accurately. For practical biosensor applications, it is important that the time required for the acquirement of the results and for biosensor regeneration is as short as possible.

The flow rate in the system was varied between 0 (stopping the flow for the measurements) and $5.1 \mathrm{~cm} / \mathrm{sec}$; at flow rates over $5.1 \mathrm{~cm} / \mathrm{sec}$ the waste of reagents became unreasonable. In case the flow rates were below $0.3 \mathrm{~cm} / \mathrm{sec}$, the experimental noise was very big due to the air bubbles, gathering on the surfaces of the sensors and walls of the flow channels and the value of the signal to noise ratio was below 3 .

\subsubsection{Sensitivity of the biosensor system based on different calibration parameters}

As described earlier, two different calibration parameters were used: the maximum signal change parameter $A$ and the apparent maximal speed parameter $v_{a p p}$, determined as described in chapter 2.3. The biosensor calibration curves were made by plotting these parameters versus glucose concentration, as presented in Fig. 6 (A and B).

The glucose assay had a linear range up to $1.2 \mathrm{mM}$; at higher glucose concentrations the dependence became nonlinear. In case the measurements were carried out with the stopped flow, the biosensor showed linearity up to $0.8 \mathrm{mM}$. The linear part of these calibration curves and the values of slopes characterize the sensitivity of the biosensing system. Due to the different nature of the used calibration parameters, the dependences of the value of their slopes on flow rate are different (Fig. 7).

The maximum signal change parameter did not substantially depend on the flow rate in the range of the studied glucose concentrations $(0.2$ to $1.5 \mathrm{mM})$. Actually, this reaction parameter is also indifferent towards the determination of time, at which the analyte front reaches 

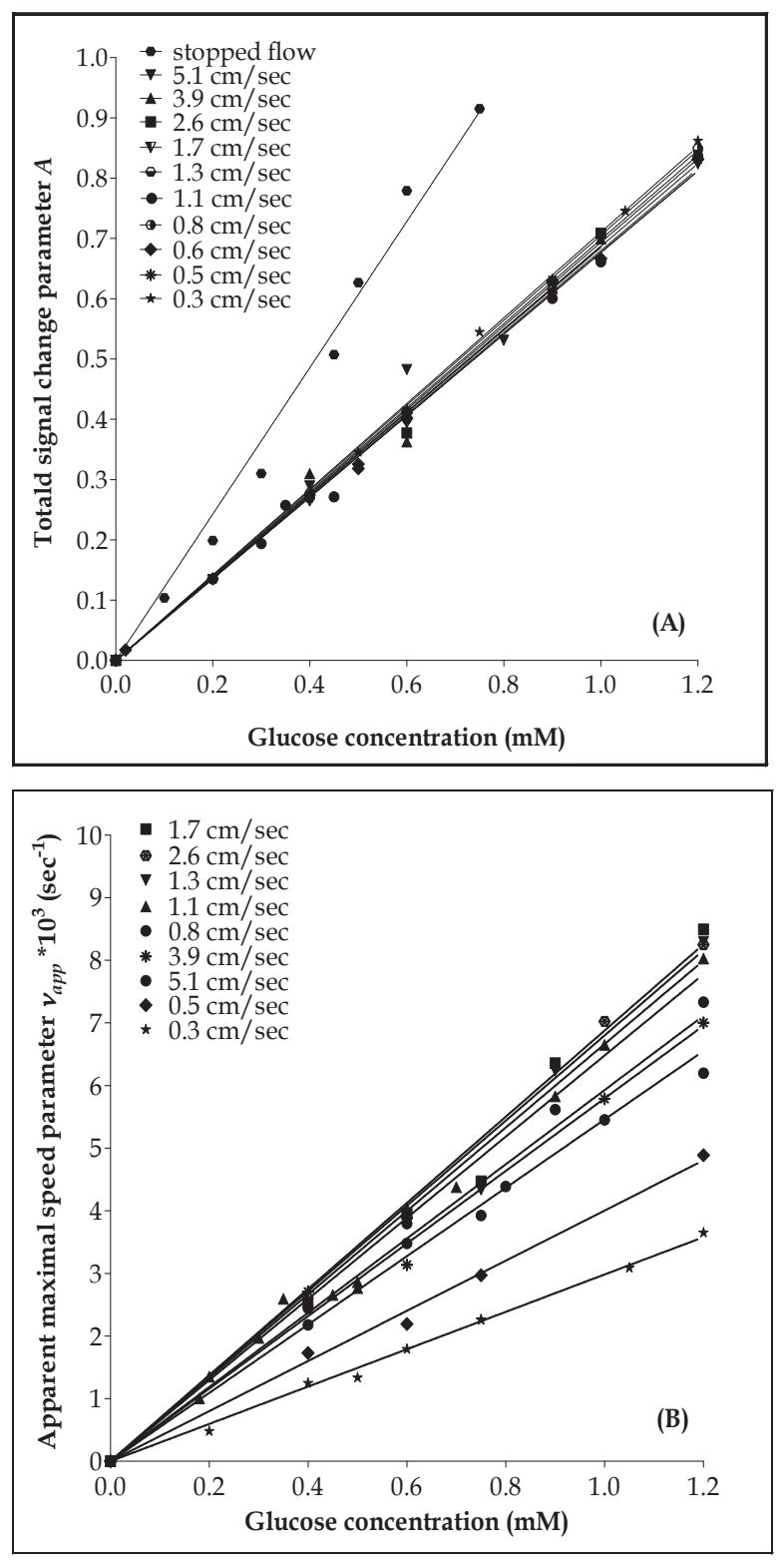

Figure 6. (A). Glucose calibration curves based on maximum signal change parameter $A$ at different flow rates. Measurements were performed at $37^{\circ} \mathrm{C}$ in a $0.1 \mathrm{M}$ phosphate buffer $(\mathrm{pH} 6.50)$. The values of all points are the results of at least 3 parallel measurements. (B). Glucose calibration curves based on the apparent maximal speed parameter $v_{a p p}$ at different flow rates. Measurements were performed at $37^{\circ} \mathrm{C}$ in a $0.1 \mathrm{M}$ phosphate buffer ( $\left.\mathrm{pH} 6.50\right)$. The values of all points are the results of at least 3 parallel measurements. 


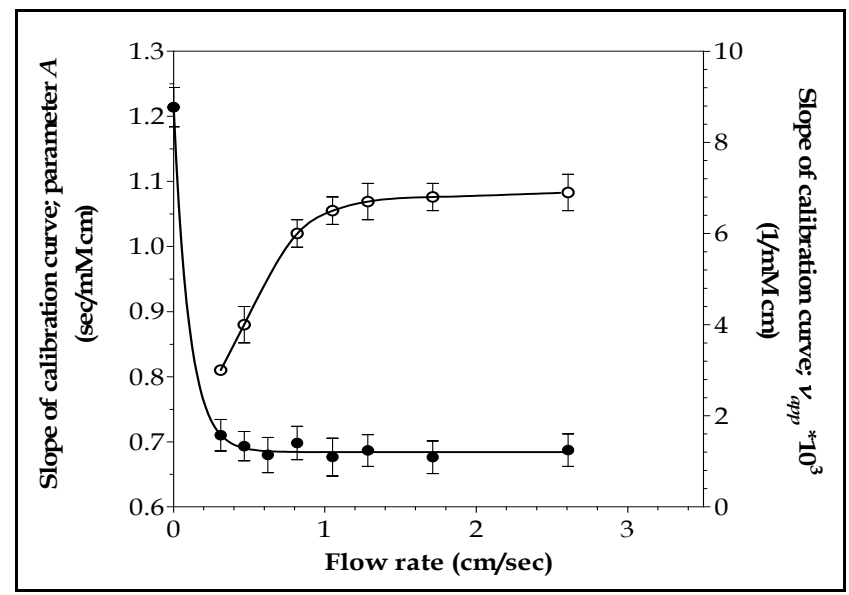

Figure 7. Dependence of the slopes of the calibration curves on different flow rates. The slopes were calculated from the calibration curves. On the left side $(\bullet)$ is the biosensor system response parameter $A$ and on the right side $(0)$ the apparent maximal speed parameter $v_{\text {app }}$.

the biosensor, as it is defined as a biosensor maximum signal change in steady-state conditions $(t \rightarrow \infty)$. In case this parameter for the glucose oxidation reaction was measured in the standing medium (the flow was stopped), the values of this parameter at different glucose concentrations were significantly higher (Fig. 6A) and the slope of the calibration curve was about 1.7 times higher than it should be, if the same signal rising mechanism had been considered. Actually this increase has only a qualitative value, as the hydraulic stroke of halting the flow influences the parameter values. Actually, in the standing medium the diffusion layer of oxygen and glucose at the surface of the sensors is much thicker and the impact of the reaction kinetics of the measured signal is much bigger than in the flowing mediums. Due to the accumulation of air bubbles in the flow system at small flow rates, it was not possible to carry out experimental measurements at flow rates under $0.8 \mathrm{~cm} / \mathrm{sec}$ and it is not clear, at which flow rates the signal rising mechanism changes.

The slope of biosensor calibration curve constructed with the apparent maximum speed parameter $v_{\text {app }}$, increases along with the increase of the flow rate until $1.1 \mathrm{~cm} / \mathrm{sec}$; at higher flow rates it reaches its maximum value and glucose calibration curves are similar. Thus, applying this parameter, the sensitivity of the biosensor can be modified according to the aim of analysis. As already pointed out, it was not possible to conduct measurements at flow rates under $0.8 \mathrm{~cm} / \mathrm{sec}$.

The flow rate also influences the biosensor response time. In standing solutions, 8 minutes were the minimal time of acquiring results with acceptable precision. So the flow rate of 1.3 $\mathrm{cm} / \mathrm{sec}$ was chosen for the studies of the system repeatability, as it offers acceptable response time and sufficient sensitivity. 
The repeatability of the experimental measurements was studied at glucose concentration of $0.5 \mathrm{mM}$ (15 experiments per day and four days in a row). The repeatability of the measurements was very good considering that the standard deviation of the vertical distances of the points from the line $S_{y . x}$. was 0.0051 and the coefficient of determination $R^{2}$ was $98 \%$ (Fig.8). The results indicated the biosensor to exhibit a fairly analytical feature of repeatability.

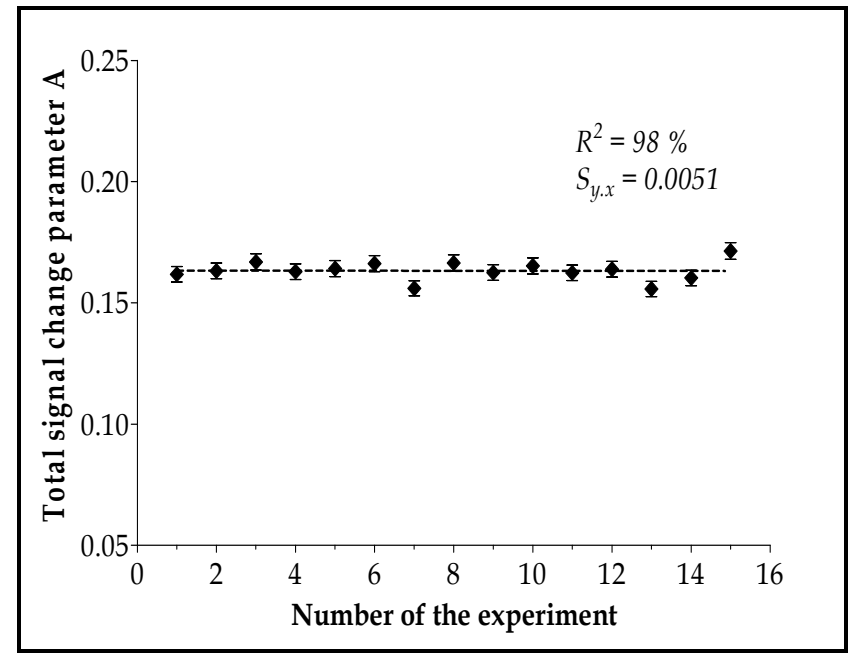

Figure 8. Repeatability of the measurements with the glucose biosensor. Measurements were carried out at $37^{\circ} \mathrm{C}$ in $0.5 \mathrm{mM}$ glucose solutions in $0.1 \mathrm{M}$ phosphate buffer $(\mathrm{pH} 6.50)$ at flow rate $1.3 \mathrm{~cm} / \mathrm{sec}$.

\subsection{Operational stability of the biosensor}

The loss of sensitivity under operational conditions is one of the most serious limits of the practical utility of biosensors. Besides possible leaching of the bio-selective material, the biosensors are ascribed to the inactivation and denaturation of their bioactive compounds. The operational stability of the present biosensor system was assessed by a continuous long-term experiment, in which we repeatedly analysed $0.5 \mathrm{mM}$ glucose solutions. The biosensor system was in everyday exploitation - used for about a 15-measurement-serie per day - after which it was washed with $0.1 \mathrm{M} \mathrm{PB}(\mathrm{pH} 6.50)$ and left overnight at $37^{\circ} \mathrm{C}$. The initial activity of the sensor dropped for about $20 \%$ during the first 3 days; after that the biosensor response remained constant for over 35 days operation period with no significant loss of activity (Fig. 9). 


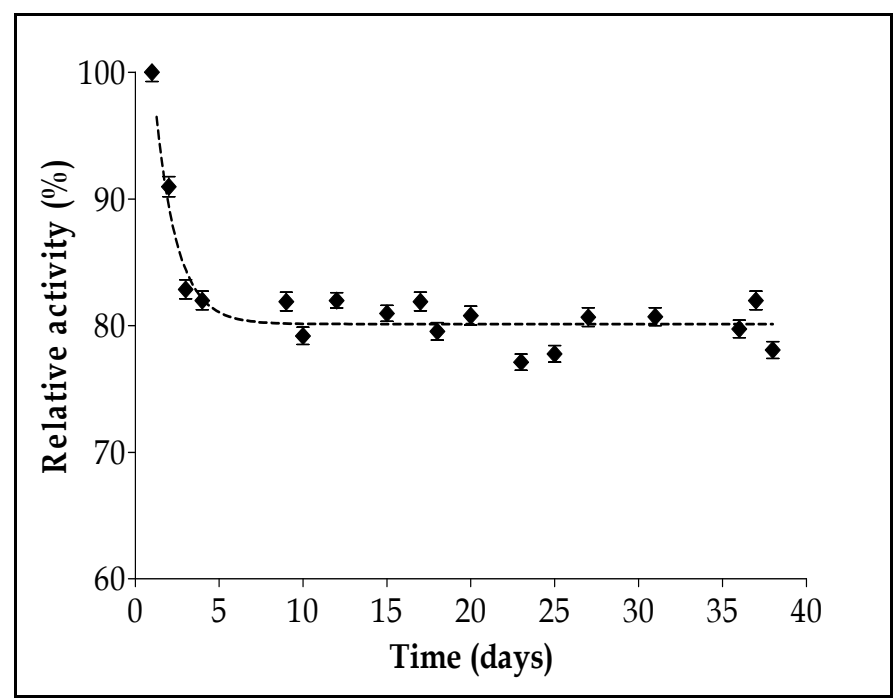

Figure 9. Stability of the biosensor with a GOD-containing nylon thread held at $37^{\circ} \mathrm{C}$. Measurements were carried out in $0.5 \mathrm{mM}$ glucose solutions in $0.1 \mathrm{M}$ phosphate buffer $(\mathrm{pH} 6.50)$ at flow rate $1.3 \mathrm{~cm} / \mathrm{sec}$. The values of all points are the results of at least 3 parallel measurements.

\section{Conclusions}

A differential optrode based biosensor system for real-time monitoring of glucose in flowthrough set-up has been studied and the selection of different calibration parameters analyzed. The influences of the flow rate and oxygen fluctuations on the sensor response have been studied. It was found that even at quite low flow rates the rising of the biosensor signal was controlled by diffusion and only in standing solutions the kinetics of the bio-recognition reaction had a substantial impact on the measurable output. The biosensor steady-state signal, calculated from the transient response was not dependent on the flow rate, if the latter exceeded $0.8 \mathrm{~cm} / \mathrm{sec}$.

The applied enzyme immobilizing procedure ensured a good operational stability of the system. Thus, an interference and cross-talk free device for the real-time monitoring of glucose concentration was successfully established. Used sensing system can be generalized for the other biologically important compounds catalyzed by oxidase-class enzymes and for the construction of biosensor arrays for different applications.

\section{List of symbols}

$c_{\mathrm{O}_{2}}(t)$ Biosensor output current at time moment $t$ 
$c_{\mathrm{O}_{2}}(0)$ Output current at the start of the reaction

$t$ Time

A Complex coefficient, corresponding to the total possible biosensor signal change at the steady-state

$B$ Initial maximal slope of process curve

$\tau_{s}$ Time constant of the transducer's response

$v_{\text {app }}$ Apparent maximal speed parameter

$S_{y \cdot x}$ Standard deviation of the vertical distances of the points from the line

\section{Acknowledgements}

This work was supported by Estonian Science Foundation grant No. 9061. Special thanks to Dr. R. Jaaniso and A. Floren for providing the oxygen optrodes and constructing the flow cell.

\section{Author details}

K. Kivirand, M. Kagan and T. Rinken

Institute of Chemistry, University of Tartu, Estonia

\section{References}

[1] Akin,M., Prediger,A., Yuksel,M., Höpfner,T., Demirkol,D.O., Beutel,S., Timur,S., \& Scheper,T (2011) A new set up for multi-analyte sensing: At-line bio-process monitoring Biosensors and Bioelectronics, 26, 4532-4537.

[2] Baker,D.A \& Gough,D.A (1996) Dynamic Delay and Maximal Dynamic Error in Continuous Biosensors Analytical Chemistry, 68, 1292-1297.

[3] Bankar,S.B., Bule,M.V., Singhal,R.S., \& Ananthanarayan,L (2009) Glucose oxidase An overview Biotechnology Advances, 27, 489-501.

[4] Baronas,D., Ivanauskas,F., \& Baronas,R (2011) Mechanisms controlling the sensitivity of amperometric biosensors in flow injection analysis systems Journal of Mathematical Chemistry, 49, 1521-1534. 
[5] Baronas,R., Ivanauskas,F., \& Kulys,J (2002) Modelling dynamics of amperometric biosensors in batch and flow injection analysis Journal of Mathematical Chemistry, 32, 225-237.

[6] Betancor,L., Lopez-Gallego,F., Hidalgo,A., Alonso-Morales,N., Mateo,G.D.O.C., Fernandez-Lafuente,R., \& Guisan,J.M (2006) Different mechanisms of protein immobilization on glutaraldehyde activated supports: Effect of support activation and immobilization conditions Enzyme and Microbial Technology, 39, 877-882.

[7] Bidmanova,S., Chaloupkova,R., Damborsky,J., \& Prokop,Z (2010) Development of an enzymatic fiber-optic biosensor for detection of halogenated hydrocarbons Analytical and Bioanalytical Chemistry, 398, 1891-1898.

[8] Cao,L (2005) Immobilised enzymes: science or art? Current Opinion in Chemical Biolo$g y, 9,217-226$.

[9] Castillo,J., Gaspar,S., Leth,S., Niculescu,M., Mortari,A., Bontidean,I., Soukharev,V., Dorneanu,S.A., Ryabov,A.D., \& Csöregi,E (2004) Biosensors for life quality - Design, development and applications Sensors and Actuators, B: Chemical, 102, 179-194.

[10] Chen,Y., Andersson,A., Mecklenburg,M., Xie,B., \& Zhou,Y (2011) Dual-signal analysis eliminates requirement for milk sample pretreatment Biosensors and Bioelectronics, $29,115-118$.

[11] D.R.Walt (2006) Fiber Optic Array Biosensors BioTechniques, 41, 529-535.

[12] Gibson,T.D (1999) Biosensors: The stability problem Analusis, 27, 630-638.

[13] Gülce,H., Ataman,I., Gülce,A., \& Yildiz,A (2002) A new amperometric enzyme electrode for galactose determination Enzyme and Microbial Technology, 30, 41-44.

[14] Hansen,E.H (1996) Principles and Applications of Flow Injection Analysis in Biosensors Journal of Molecular Recognition, 9, 316-325.

[15] Hartwell,S.K \& Grudpan,K (2012) Flow-Based Systems for Rapid and High-Precision Enzyme Kinetics Studies Journal of Analytical Methods in Chemistry, 2012.

[16] Hu,N., Zhao,M.H., Chuang,J.L., \& Li,L (2011) The application study of biosensors in environmental monitoring Cross Strait Quad-Regional Radio Science and Wireless Technology Conference, 2, 976-979.

[17] Isgrove,F.H., Williams,R.J.H., Niven,G.W., \& Andrews,A.T (2001) Enzyme immobilization on nylon-optimization and the steps used to prevent enzyme leakage from the support Enzyme and Microbial Technology, 28, 225-232.

[18] Ivanauskas,F \& Baronas,R (2008) Modelling an amperometric biosensor acting in a flowing liquid International Journal for Numerical Methods in Fluids, 56, 1313-1319.

[19] Jaaniso,R., Avarmaa,T., Suisalu,A., Floren,A., Ruudi,A., \& Õige,K (2005) Stability of luminescence decay parameters in oxygen sensitive polymer films doped with Pdporphyrins Optical Materials and Applications, Proceedings of SPIE, 5946, 1-10. 
[20] Jia,N.Q., Zhang,Z.R., Zhu,J.Z., \& Zhang,G.X (2004) Multianalyte biosensors for the simultaneous determination of glucose and galactose based on thin film electrodes Chinese Chemical Letters, 15, 322-325.

[21] Kivirand,K., Rebane,R., \& Rinken,T (2011) A simple biosensor for biogenic diamines, comprising amine oxidase - Containing threads and oxygen sensor Sensor Letters, 9, 1794-1800.

[22] Kivirand,K \& Rinken,T (2009) Preparation and Characterization of Cadaverine Sensitive Nylon Thread Sensor Letters, 7, 580-585.

[23] Kivirand,K \& Rinken,T (2011) Biosensors for Biogenic Amines: The Present State of Art Mini-Review Analytical Letters, 44, 2821-2833.

[24] Konermann,L (1999) Monitoring Reaction Kinetics in Solution by Continuous-Flow Methods: The Effects of Convection and Molecular Diffusion under Laminar Flow Conditions Journal of Physical Chemistry A, 103, 7210-7216.

[25] Lammertyn,J., Verboven,P., Veraverbeke,E.A., Vermeir,S., Irudayaraj,J., \& Nicolai,B.M (2006) Analysis of fluid flow and reaction kinetics in a flow injection analysis biosensor Sensors and Actuators, B: Chemical, 114, 728-736.

[26] Leung,A., Shankar,P.M., \& Mutharasan,R (2007) A review of fiber-optic biosensors Sensors and Actuators B: Chemical, 125, 688-703.

[27] Li,L \& Walt,D.R (1995) Dual-analyte fiber-optic sensor for the simultaneous and continuous measurement of glucose and oxygen Analytical Chemistry, 67, 3746-3752.

[28] Maestre,E., Katakis,I., Narvez,A., \& Dominguez,E (2005) A multianalyte flow electrochemical cell: Application to the simultaneous determination of carbohydrates based on bioelectrocatalytic detection Biosensors and Bioelectronics, 21, 774-781.

[29] Marazuela,M.D \& Moreno-Bondi,M.C (2002) Fiber-optic biosensors - An overview Analytical and Bioanalytical Chemistry, 372, 664-682.

[30] Mateo,C., Palomo,J.M., Fernandez-Lorente,G., Guisan,J.M., \& Fernandez-Lafuente,R (2007) Improvement of enzyme activity, stability and selectivity via immobilization techniques Enzyme and Microbial Technology, 40, 1451-1463.

[31] Mehrvar,M \& Abdi,M (2004) Recent developments, characteristics, and potential applications of electrochemical biosensors Analytical Science, 20, 1113-1126.

[32] Mello,L.D \& Kubota,L.T (2002) Review of the use of biosensors as analytical tools in the food and drink industries Food Chemistry, 77, 237-256.

[33] Mishra,R.K., Dominguez,R.B., Bhand,S., Munoz,R., \& Marty,J.L (2012) A novel automated flow-based biosensor for the determination of organophosphate pesticides in milk Biosensors and Bioelectronics, 32, 56-61. 
[34] Nan,C., Zhang,Y., Zhang,G., Dong,C., Shuang,S., \& Choi,M.M.F (2009) Activation of nylon net and its application to a biosensor for determination of glucose in human serum Enzyme and Microbial Technology, 44, 249-253.

[35] Õige,K., Avarmaa,T., Suisalu,A., \& Jaaniso,R (2005) Effect of long-term aging on oxygen sensitivity of luminescent Pd-tetraphenylporphyrin/PMMA films Sensors and Actuators, B: Chemical, 106, 424-430.

[36] Pahujani,S., Kanwar,S.S., Chauhan,G., \& Gupta,R (2008) Glutaraldehyde activation of polymer Nylon-6 for lipase immobilization: Enzyme characteristics and stability Bioresource Technology, 99, 2566-2570.

[37] Palmisano,F., Rizzi,R., Centonze,D., \& Zambonin,P.G (2000) Simultaneous monitoring of glucose and lactate by an interference and cross-talk free dual electrode amperometric biosensor based on electropolymerized thin films Biosensors and Bioelectronics, 15, 531-539.

[38] Pasic,A., Koehler,H., Klimant,I., \& Schaupp,L (2007) Miniaturized fiber-optic hybrid sensor for continuous glucose monitoring in subcutaneous tissue Sensors and Actuators, B: Chemical, 122, 60-68.

[39] Pasic,A., Koehler,H., Schaupp,L., Pieber,T.R., \& Klimant,I (2006) Fiber-optic flowthrough sensor for online monitoring of glucose Analytical and Bioanalytical Chemistry, 386, 1293-1302.

[40] Peedel,D \& Rinken,T (2012) Effect of Temperature on the Catalytic Properties of Enzymes, Used in Lactose Cascade Biosensors and the Sensitivity of Lactose Biosensing System Proceedings of the Estonian Academy of Sciences Article in press.

[41] Polster,J., Prestel,G., Wollenweber,M., Kraus,G., \& Gauglitz,G (1995) Simultaneous determination of penicillin and ampicillin by spectral fibre-optical enzyme optodes and multivariate data analysis based on transient signals obtained by flow injection analysis Talanta, 42, 2065-2072.

[42] Reder-Christ,K \& Bendas,G (2011) Biosensor applications in the field of antibiotic research-a review of recent developments Sensors, 11, 9450-9466.

[43] Rinken,T \& Tenno,T (2001) The dynamic signal lag of amperometric biosensors Characterisation of glucose biosensor output Biosensors and Bioelectronics, 16, 53-59.

[44] Sarma,A.K., Vatsyayan,P., Goswami,P., \& Minteer,S.D (2009) Recent advances in material science for developing enzyme electrodes Biosensors and Bioelectronics, 24, 2313-2322.

[45] Sassolas,A., Blum,L.J., \& Leca-Bouvier,B.D (2012) Immobilization strategies to develop enzymatic biosensors Biotechnology Advances, 30, 489-511.

[46] Segura-Ceniceros,E.P., Dabek,K.R., \& Ilyina,A.D (2006) Invertase immobilization on nylon- 6 activated by hydrochloric acid in the presence of glutaraldehyde as crosslinker Vestnik Moskovskogo Universiteta, Seriya 2: Khimii, 47, 143-148 
[47] Surareungchai,W., Worasing,S., Sritongkum,P., Tanticharoen,M., \& Kirtikara,K (1999) Dual electrode signal-subtracted biosensor for simultaneous flow injection determination of sucrose and glucose Analytica Chimica Acta, 380, 7-15.

[48] Thevenot,D.R., Toth,K., Durst,R.A., \& Wilson,G.S (2001) Electrochemical biosensors: Recommended definitions and classification Biosensors and Bioelectronics, 16, 121-131.

[49] Tsai,H.c \& Doong,R (2005) Simultaneous determination of pH, urea, acetylcholine and heavy metals using array-based enzymatic optical biosensor Biosensors and Bioelectronics, 20, 1796-1804.

[50] Viveros,L., Paliwal,S., McCrae,D., Wild,J., \& Simonian,A (2006) A fluorescence-based biosensor for the detection of organophosphate pesticides and chemical warfare agents Sensors and Actuators B: Chemical, 115, 150-157.

[51] Yang,C., Zhang,Z., Shi,Z., Xue,P., Chang,P., \& Yan,R (2010) Application of a novel co-enzyme reactor in chemiluminescence flow-through biosensor for determination of lactose Talanta, 82, 319-324.

[52] Zhou,X., Medhekar,R., \& Toney,M.D (2003) A continuous-flow system for high-precision kinetics using small volumes Analytical Chemistry, 75, 3681-3687.

[53] Zhu,L., Li,Y., \& Zhu,G (2002) A novel flow through optical fiber biosensor for glucose based on luminol electrochemiluminescence Sensors and Actuators B: Chemical, 86, 209-214. 
